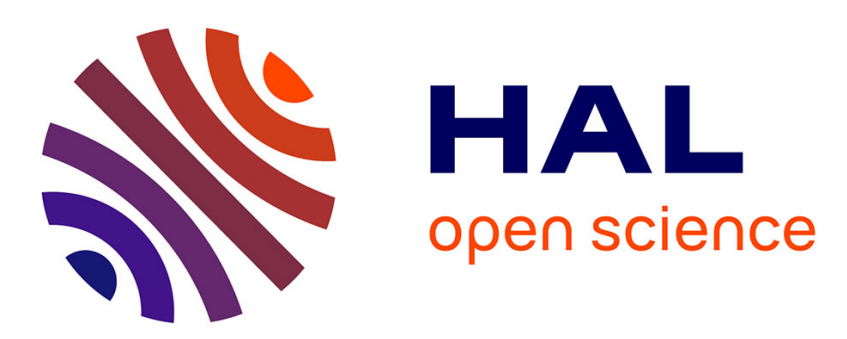

\title{
A Vignetting Model for Light Field Cameras with an Application to Light Field Microscopy
}

Lois Mignard-Debise, Ivo Ihrke

\section{To cite this version:}

Lois Mignard-Debise, Ivo Ihrke. A Vignetting Model for Light Field Cameras with an Application to Light Field Microscopy. IEEE Transactions on Computational Imaging, 2019, pp.10. 10.1109/TCI.2019.2911856 . hal-02263377

\section{HAL Id: hal-02263377 \\ https://hal.science/hal-02263377}

Submitted on 5 Aug 2019

HAL is a multi-disciplinary open access archive for the deposit and dissemination of scientific research documents, whether they are published or not. The documents may come from teaching and research institutions in France or abroad, or from public or private research centers.
L'archive ouverte pluridisciplinaire HAL, est destinée au dépôt et à la diffusion de documents scientifiques de niveau recherche, publiés ou non, émanant des établissements d'enseignement et de recherche français ou étrangers, des laboratoires publics ou privés. 


\title{
A Vignetting Model for Light Field Cameras with an Application to Light Field Microscopy
}

\author{
Loïs Mignard-Debise and Ivo Ihrke, \\ independent scholars
}

\begin{abstract}
In standard photography, vignetting is considered mainly as a radiometric effect because it results in a darkening of the edges of the captured image. In this paper, we demonstrate that for light field cameras, vignetting is more than just a radiometric effect. It modifies the properties of the acquired light field and renders most of the calibration procedures from the literature inadequate. We address the problem by describing a modeland camera-agnostic method to evaluate vignetting in phase space. This enables the synthesis of vignetted pixel values, that, applied to a range of pixels yield images corresponding to the white images that are customarily recorded for calibrating light field cameras. We show that the commonly assumed reference points for microlens-based systems are incorrect approximations to the true optical reference, i.e. the image of the center of the exit pupil. We introduce a novel calibration procedure to determine this optically correct reference point from experimental white images. We describe the changes vignetting imposes on the light field sampling patterns and, therefore, the optical properties of the corresponding virtual cameras using the ECA model [1] and apply these insights to a custom-built light field microscope.
\end{abstract}

Index Terms - Light field, light field camera, optics, vignetting, calibration.

\section{INTRODUCTION \& RELATED WORKS}

$\mathbf{T}$ HE field of light field imaging has seen the emergence of many different types of cameras to measure the light field of a scene. The model behind their design is most of the time a first-order optical model, employing a single ray per pixel and ignoring the finite extent of the optical components that constitute the optical system. These models therefore omit vignetting effects that occur when finite ray bundles are partially or fully blocked by the various mounts and the finite extent of the lenses during their propagation into the camera.

However, as we show in this paper, these vignetting effects are crucial to the comprehension and design of proper light field cameras as they influence the optical properties of the captured light field as well as the calibration procedure. Overall, the contributions of this paper are:

- a characterization of vignetting in light field cameras that is independent of the camera model and light field system being analyzed,

- a study of white image generation, including the practically observed cat's eye shape, using the above model exemplified for microlens array (MLA) based light field cameras,

- the identification of the optically correct reference point in microlens based light field cameras, and a novel calibration procedure exploiting these insights, and
- as an application of the developed techniques, an evaluation of the effect on equivalent camera array [1] properties in a case study involving a custom-built light field microscope.

In the following, we discuss the areas of related work relevant to this paper.

a) Light Field Systems: We develop our vignetting analysis in phase space, Sect. II The vignetting model itself can be applied to any kind of light field imaging system, but we later exemplify its effect on the most commonly used MLA-based light field cameras.

These cameras are based on the "integral imaging" technique of Lippmann [2], which has been reinvented [3] and been made practical with the advent of digital imaging. The two main variants are the "afocal" [4] and the "focused" [5] configuration, depending on the microlens to sensor distance.

Light fields can also be captured by camera arrays [6], [7], [8], [9]. However, these are not affected by the effect discussed in this paper since the cameras are not virtual.

A variety of other optically implemented light field camera designs exist that, in principle, are subject to the vignetting effect discussed here since they also have a virtual camera description [1] that is affected by our analysis: External lens arrays placed before the camera achieve a large baseline [10], [11], [12], while mirror arrays are a good way to cover a large field of view [13], [14], [15], [16]. The number of virtual viewpoints in mirror systems can be augmented considerably by utilizing inter-reflections between mirrors [17], [18]. Kaleidoscopic light field imaging [19], [20] uses a light pipe inside a camera for this purpose. While we only show the effects for MLA-based systems in a case study involving a light field microscope [21], Sect. IV] these alternative systems should be checked in future work.

b) Light Field Design Criteria: Vignetting is an essential part of MLA-based light field camera design since it prevents light leakage between the different sub-images that manifests itself as cross-talk or ghosting. For microlens based light field cameras, this task is addressed using the f-number matching rule [4], [22]. It states that the working f-number of the microlenses and that of the main objective should be equal to have pixels receiving light from a single microlens only and thus avoid cross-talk. However, this important rule ignores the effect of other apertures in the system that may (partially or fully) block the ray bundles and modify the light distribution in the sub-images. Our paper improves on this aspect of light field system design. 
c) Optical Models: The operating principle of light field cameras is today primarily modeled with first-order ray-based models. For microlens based light field cameras, [23], [24] provide equations and a deeper understanding of the optics at play in these systems which enables better performance for the rendering techniques used to synthesize a viewpoint at high resolution.

The sampling pattern cube (SPC) model is a more general framework [25] that can be applied to different types of light field cameras. It divides the light field sampling performed by the camera into a series of elementary sampling structures (light pyramids) and maps them to the object space of the camera. The model evaluates the system properties such as lateral resolution in this space accounting for the size of pixels and the vignetting by the system apertures. The model only applies to single pixels and is targeted at understanding object space properties of the corresponding ray bundles.

The equivalent camera array (ECA) model [1] has a similar approach but it decomposes the system into a more accurate set of elementary sampling structures (pairs of apertures) that are also imaged to the object space. In contrast to the SPC model, the ECA model reorganizes these structures into a higher level representation: a virtual camera array (in object space) and it derives the optical properties of these virtual cameras. It is therefore well suited to compare widely different light field camera designs.

However, the ECA model assumes optical components with infinite apertures. In Sect. IV, we apply this model in the context of vignetting in a light field microscope involving relay optics and explore the significant consequences on the properties of the virtual cameras.

d) Calibration: The calibration task for a light field camera is essential to extract the light field data from the raw image given by the sensor. It first consists in associating a light ray to each pixel to account for misalignment between the optical components [26], [27] and to parameterize the light field data. This step necessitates the identification of a reference point for each micro-image. This reference point is known to suffer from bias at the image boundaries [28], [29], [30] due to vignetting. We show that this effect is caused by incorrect assumptions on the position of the reference point.

Our vignetting model simulates the light distribution on the raw image which is necessary for a correct calibration. We define the optically appropriate reference point also for the case of vignetted micro-images at the image boundary.

\section{Vignetting Model for Light Field Cameras}

In this section, we propose a method to determine the amount of vignetting of a first order optical system based on the evaluation of its etendue in phase space. This model does not rely on any specific light field camera model. Then, we use this method in Sect. III to simulate the irradiance on the sensor of MLA-based light field cameras and we discuss the true reference positions for the sensor sub-images to correctly extract the light field.

\section{A. Vignetting Model}

Phase space is a practical way to represent ray bundles. In this space, a ray is defined as a point, we denote its light field coordinates at the phase space evaluation plane $i$ as $\mathbf{p}_{i}=$ $(u, v, s, t)$. Let us define an indicator function $\chi$ over this phase space that associates a binary value for each ray, 1 if it is part of the ray bundle and 0 if it is not. The bundle of rays defined by $\chi$ is therefore the set $\mathcal{R}=\{\mathbf{p} \mid \chi(\mathbf{p})=1\}$.

a) Direct model: The straightforward and intuitive method to compute vignetting is to sample rays from the pixels and propagate them through the system to the objectspace, verifying whether they encounter a surface during their travel, see Fig. 1a Since we consider that the deflection of rays follows the equations of the paraxial regime, optical elements can be reduced to a simple form: an aperture of finite size in the plane of refraction or reflection. As such, a ray $\mathbf{p}$ can be affected by two different transformations from $\mathbb{R}^{4}$ to $\mathbb{R}^{4}$. The first is the free space transport $t_{i \rightarrow j}$ from plane $i$ to plane $j$. The second is the deviation of the ray at plane $i$ either by reflection of refraction that we denote $r_{i}$. Consider an optical system with $N$ optical elements that we index starting from the sensor with index 0 to object-space. The blocking of rays by an aperture at surface $i$ is described by the following function:

$$
\chi_{i}^{S}\left(\mathbf{p}_{i}\right)= \begin{cases}1 & \text { if }(u, v) \text { is in the area of aperture } \mathrm{i}, \\ 0 & \text { otherwise }\end{cases}
$$

For instance, the ray bundle that hits a pixel in the sensor plane 0 in the absence of any other obstructing components is then defined by the aperture function $\chi_{0}^{S}\left(\mathbf{p}_{0}\right)$.

The ray bundle at surface $i$ before and after deflection by the optical element is described by two functions, one before and one after the ray deviation, respectively. We denote these functions by $\chi_{i}^{r}\left(\mathbf{p}_{i}\right)$ and $\widehat{\chi}_{i}^{r}\left(\widehat{\mathbf{p}}_{i}\right)$. They are related by

$$
\widehat{\chi}_{i}^{r}\left(\widehat{\mathbf{p}}_{i}\right)=\chi_{i}^{r}\left(r^{-1}\left(\widehat{\mathbf{p}}_{i}\right)\right) .
$$

For apertures with no optical effect, we have directly $\widehat{\chi}_{i}^{r}\left(\widehat{\mathbf{p}}_{i}\right)=$ $\chi_{i}^{r}\left(\widehat{\mathbf{p}}_{i}\right)$. Propagating the ray bundle through the system consists in finding the consecutive $\widehat{\chi}_{i}^{r}$ of each surface $i$. For the case of a pixel, $\widehat{\chi}_{0}^{r}=\chi_{0}^{S}$ since we consider it as an aperture without optical effect. The ray bundle hitting the pixel after passing through aperture 1 is defined before deviation by

$$
\chi_{1}^{r}\left(\mathbf{p}_{1}\right)=\chi_{0}^{S}\left(t_{0 \rightarrow 1}^{-1}\left(\mathbf{p}_{1}\right)\right) \cap \chi_{1}^{S}\left(\mathbf{p}_{1}\right)
$$

and, after deviation by

$$
\begin{aligned}
\widehat{\chi}_{1}^{r}\left(\widehat{\mathbf{p}}_{1}\right) & =\chi_{1}^{r}\left(r^{-1}\left(\widehat{\mathbf{p}}_{1}\right)\right) \\
& =\chi_{0}^{S}\left(t_{0 \rightarrow 1}^{-1}\left(r_{1}^{-1}\left(\widehat{\mathbf{p}}_{1}\right)\right) \cap \chi_{1}^{S}\left(r_{1}^{-1}\left(\widehat{\mathbf{p}}_{1}\right)\right)\right. \\
& =\widehat{\chi}_{0}^{r}\left(t_{0 \rightarrow 1}^{-1}\left(r_{1}^{-1}\left(\widehat{\mathbf{p}}_{1}\right)\right) \cap \chi_{1}^{S}\left(r_{1}^{-1}\left(\widehat{\mathbf{p}}_{1}\right)\right) .\right.
\end{aligned}
$$

For the following surfaces, we have

$\widehat{\chi}_{i+1}^{r}\left(\widehat{\mathbf{p}}_{i+1}\right)=\widehat{\chi}_{i}^{r}\left(t_{i \rightarrow i+1}^{-1} \circ r_{i+1}^{-1}\left(\widehat{\mathbf{p}}_{i+1}\right)\right) \cap \chi_{i+1}^{S}\left(r_{i+1}^{-1}\left(\widehat{\mathbf{p}}_{i+1}\right)\right)$.

We continue this recursion till reaching the object-space after aperture $N$. The ray bundle in object space is then described by $\widehat{\chi}_{N}^{r}$. 


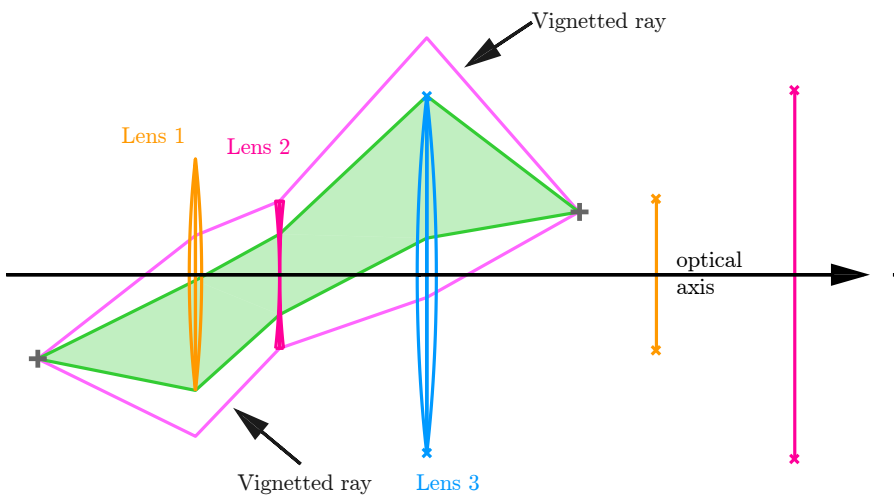

(a) Direct model

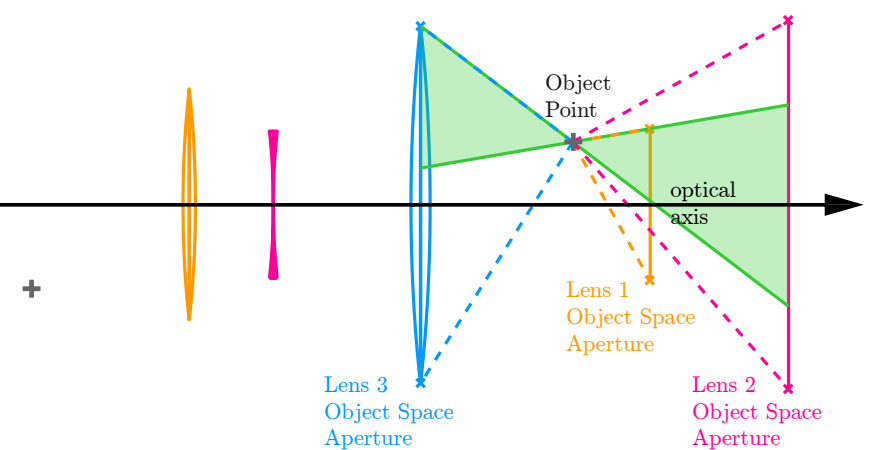

(b) Object-space aperture model

Fig. 1. Ray diagram of the vignetting models. Lens 2 is the stop of the system. (a) The pink ray bundle passes through the full stop. It is limited by the bottom of the aperture of lens 1 and by the top of the aperture of lens 3 . The green ray bundle is the largest unvignetted ray bundle. (b) Tracing lines from the object point to the boundaries of the apertures mapped into object space visually indicates which apertures affect vignetting. This method is used to determine the entrance and exit pupils and windows of an optical system [31]. Note that the resulting object space ray bundles are equivalent.

This final function is ultimately a complex recursive function that goes back to the set intersection of $N+1$ appropriately warped apertures, i.e. the optical and the pixel apertures $\chi_{i}^{S}$. Moreover, this is still a simple case since we have assumed that the plane of the aperture and the plane of ray deviation of an optical element were the same. Better models of components in the paraxial regime assume some optical thickness between the principal planes where the deviation occurs. The aperture plane can also lie in a third different plane. Consequently, the corresponding recursive equation is even more complex in practice. A simpler formulation can be obtained by working in object space.

b) Object-space model: The ray bundle that results in object-space is defined by the apertures of the system even with more complicated models for optical components. So, we can remove the ray deviations caused by the optical components on the apertures by imaging all the apertures to object-space first and computing the intersection afterwards, see Fig. $1 \mathrm{~b}$ We denote $\chi_{i}^{S_{o}}$ the function associated to the object-space image obtained after imaging aperture $i$ through the lenses $i$ to $N$ using the techniques described in [32]. Note that, in general, the phase space underlying $\chi_{i}^{S_{o}}$ will be associated with different evaluation planes $i$. In object-space, the final function is then written more simply as

$$
\widehat{\chi}_{N}^{r}\left(\widehat{\mathbf{p}}_{o}\right)=\bigcap_{i=0}^{N} \chi_{i}^{S o}\left(t_{i \rightarrow o}^{-1}\left(\widehat{\mathbf{p}}_{o}\right)\right) .
$$

where $o$ is an arbitrary common phase space evaluation plane. This rewrite not only results in simpler equations, but also in computational advantages when vignetting is computed for many pixels.

\section{B. Towards a sensor value for white images}

We now make the connection between the indicator function and the pixel irradiance. The etendue of a pixel is defined as the product of its area and the solid angle subtended by the exit pupil of the optical system [33]. Consequently, its units are square meter times steradian $\left(m^{2} \cdot s r\right)$.
The etendue $G$ of a more general ray bundle is defined as the integral of its indicator function $\chi$ :

$$
G=\int_{\mathbb{R}^{4}} \chi(\mathbf{p}) \cos ^{4} \theta(\mathbf{p}) d \mathbf{p}
$$

where $\theta$ is the angle between the pixel normal and the incoming ray described by $\mathbf{p}$. The factor $\cos ^{4} \theta$ enters as the Jacobian determinant of the reparametrization from optical phase space to the $(u, v, s, t)$ parametrization that we use in this paper.

Let's consider a representative ray in the bundle, for instance, the chief ray of a conventional optical system, or the ray passing through the centers of the pixel and the microlens for MLA-based light field cameras. We denote its radiance $L$ in watt per square meter per steradian $\left(W \cdot m^{-2} \cdot s r^{-1}\right)$. Then, the radiant flux $\Phi[W]$ of the ray bundle can be approximated as

$$
\Phi=G \cdot L
$$

Thus, the etendue is proportional to the irradiance $E=\frac{\Phi}{A}$ received by the pixel to the zeroth order, with $A$ being the (constant) pixel area. Consequently, we can use the phase space volume of a ray bundle from Equation 7 as a measure of irradiance.

Thus, we have

$$
I_{S} \approx G
$$

where $G$ is evaluated using Equation 7 with $\chi=\widehat{\chi}_{N}^{r}$ and $\cos \theta$ is computed for the representative ray only.

In practice, we estimate the phase space volume via Monte Carlo sampling or exact intersection. In the latter case, it is convenient to consider only convex aperture shapes since, in this case, the set intersection of Equation 6 is also convex meaning that convex hull algorithms can be applied. For computational efficiency, it is advisable to work with bounding boxes, obtaining computationally inexpensive answers for the fully vignetted case $G=0$ and the fully unvignetted case $G=G_{\max }$. 


\section{The MLA White Image And the Definition of THE Sub-IMAge REFERENCE POINT}

In traditional photography or in microscopy, the picture of a white Lambertian scene reveals the effects of the mechanical and natural vignetting. In light field cameras, a picture in these conditions is necessary for calibration purposes. It is used to retrieve the parametrization of the $4 \mathrm{D}$ light field from the $2 \mathrm{D}$ sensor. The goal is to associate the correct angular and spatial parameters to each pixel for further analysis and reconstruction of the light field. In this section, we demonstrate the use of our vignetting model to synthesize the white image of a MLAbased light field camera. We also point out the limitations of the calibration approaches from the literature in detecting the true reference point for the light field parametrization. Finally, we propose a new reference point detection algorithm.

\section{A. MLA White Image Synthesis}

Our vignetting model allows to simulate the calibration image (or flat field image) of a light field camera from the computation of the etendue for each sensor pixel/microlens pair using Equation 9. However, the optical system of a light field camera produces multiple images on the sensor from different light paths. For further illustration, we take the case of MLA-based light field cameras as an example. In this case, these light paths correspond to the imaging through each microlens. Depending on the optical settings, these images may overlap each other so the final intensity of one pixel has to be integrated over all lenslets. The etendue of one pixel is then the sum of the etendue over all lenslets

$$
G(\text { pixel })=\sum_{\text {lenslet }} G_{\text {lenslet }}(\text { pixel }) \text {. }
$$

In practice, though, only neighboring lenslets of a pixel need to be considered. A good design for an MLA-based light field camera follows the f-number rule [4], [22] which states that the f-number of the main lens should match that of the lenslets to avoid overlapping and its opposite, an insufficient fill-factor. Using the mockup MLA-based system of Fig. 2a we have computed its white image in Fig. 3 to discuss the current practice in light field calibration, to define the proper centers of reference and to propose our own center detection method. This optical system is made of a sensor, a MLA and a main objective with two lenses and a diaphragm. Their parameters are found in Table II. We have exaggerated the optical parameters of the system to better show the effects of vignetting on the white image such as the characteristic asymetric cat's eye shape of the sub-images at the boundaries and their intensity distribution.

More generally, the calibration of a light field camera depends on its optical settings. In the Lytro camera, for instance, a database of white images for many different zoom and focus configurations is stored inside the camera and matched to the actual settings of the acquired pictures.

\section{B. The MLA Sub-Image Reference Point}

Finding the sub-image reference point of each sub-image is the primary task of light field camera calibration as it serves
TABLE I

PARAMETERS FOR THE COMPONENTS OF THE MOCKUP SYSTEM. THE Z-POSITION IS THE POSITION ALONG THE OPTICAL AXIS, THE ORIGIN IS SET IN THE PLANE OF THE MLA. VALUES ARE IN MILLIMETERS.

\begin{tabular}{|l|c|c|c|c|}
\hline Components & Z-position & $\begin{array}{c}\text { Focal } \\
\text { length }\end{array}$ & $\begin{array}{c}\text { Aperture } \\
\text { width }\end{array}$ & $\begin{array}{c}\text { Number of } \\
\text { elements }\end{array}$ \\
\hline Sensor & -0.52 & - & 0.00125 & $119 \times 51$ \\
MLA & 0.0 & 0.40 & 0.175 & $7 \times 3$ \\
Lens 1 & 3.0 & 2.0 & 1.0 & 1 \\
Diaphragm & 4.0 & - & 0.80 & 1 \\
Lens 2 & 5.0 & 2.0 & 1.0 & 1 \\
\hline
\end{tabular}

to establish the light field parametrization: the assignment of pixel coordinates to light field coordinates. In practice, the optical system parameters of a light field camera are often not known and techniques have been developed to obtain the sub-image reference point from experimentally acquired white images.

All detection techniques estimate the reference point as the brightest point (BP) (or local maxima) of the sub-image because the brightest point lies at the orthogonal projection (OP) of the microlens center onto the sensor. Indeed, this is true as the pixel intensity $I_{S}$ in Equation 9 is maximum when the incident chief ray is normal to the pixel plane $(\theta=0)$.

There exist a variety of detection algorithms for this center estimation. A low-pass filter is performed on the image to remove noise prior to the detection [28]. The sub image is eroded and then fitted with a paraboloid, the maximum of which is used as the reference point [26]. A comparison of these two methods based on a set of synthetic data which ignores the effect of mechanical vignetting is presented in [34] where also propose two additional variants yielding improved results are proposed. The sub-image centroid is used in [35]. It is computed from a weighted average (WA) of the pixels intensities arguing that the sub-images are approximately circular.

\section{The Optical Reference Point}

The main problem with these methods is that their assumption that the brightest point of the sub-image is the true reference point is incorrect in general. The ground truth point (GT) corresponds to the projection of the center of the exit pupil of the system through the microlens center which is also the central view of the camera [29]. In [27], in order to find the ground truth, the authors close down the main lens aperture, reducing the sub-image extent to nearly a point and then perform a centroid estimation to obtain the center position with sub-pixel precision.

It is only when the pupil is at infinity that the ground truth point (GT) agrees with the orthogonally projected microlens center (OP). In this case, the brightest point estimate (BP) coincides with the ground truth. In the aforementioned literature, this configuration is closely met due to the small size and focal length of the microlenses compared to the distance of the exit pupil of the main lens.

Any modification of the position of the exit pupil modifies both the ground truth reference point (GT) and the extent of the sub-images. With an exit pupil closer to the microlens array, the micro-images become larger and the ground truth 


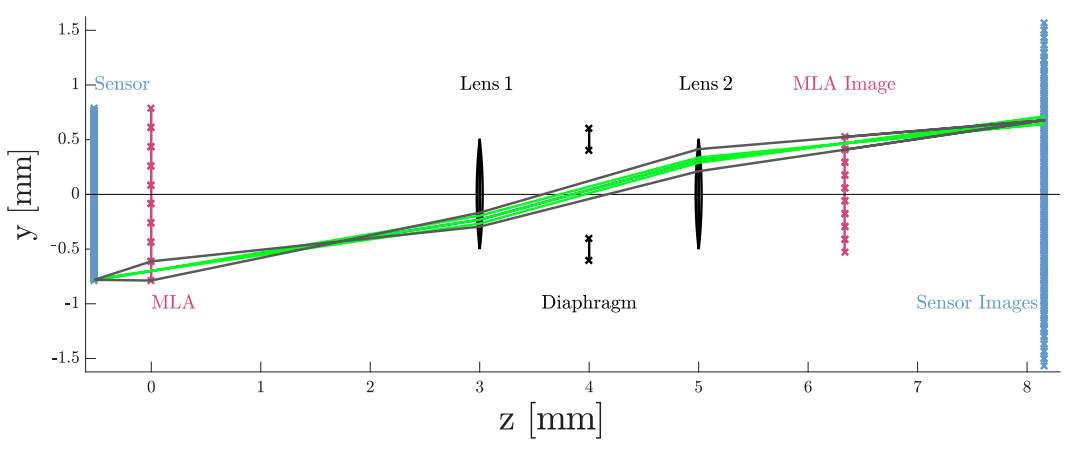

(a) Setup

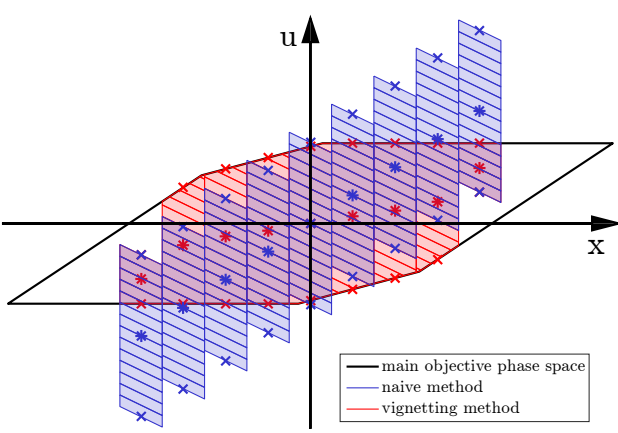

(b) Phase space

Fig. 2. Vignetting in a light field camera. (a) Setup of a light field camera. The main objective consists of two lenses and a diaphragm. Grey and green rays originate from the center of a pixel and a microlens, respectively. (b) Phase space with the unvignetted ECA model in blue and the method using our vignetting model in red. The $*$ markers indicate the view directions and the $\times$ markers indicate the limits of the angle of view of each virtual camera. The black polygon delimits the phase space region of the main objective of the camera.

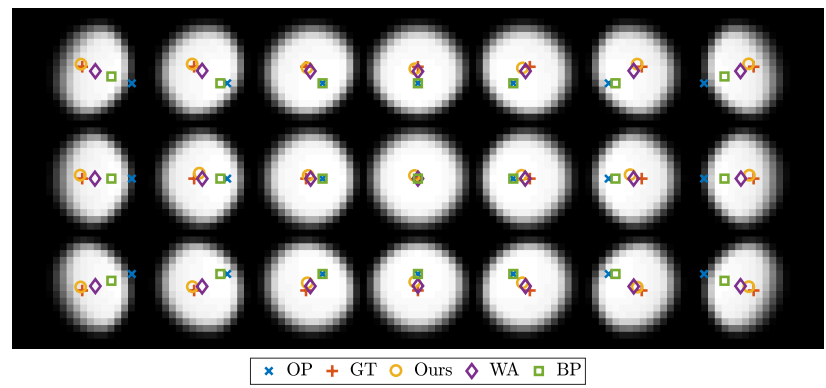

(a) White Image

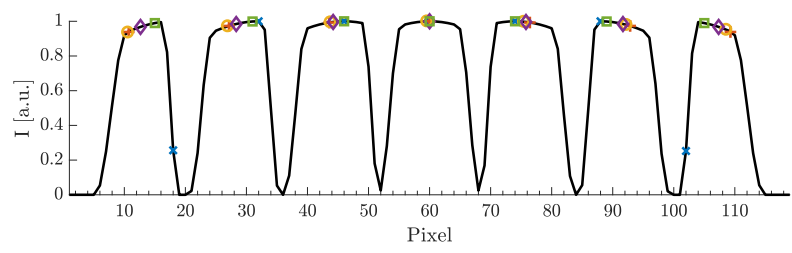

(b) Intensity profile of the center row

Fig. 3. White image of the system from Fig. 2 The intensity maximum of a sub-image corresponds to the orthogonal projection of the microlens center and is considered as the reference point for light field parametrization. We argument that the true reference point is the perspective projection of the system pupil. We propose a method based on a circle fit of the sub-images the center of which matches the true reference better.

points are more widely spaced. Considering that the sensor parameters are fixed, this implies that fewer micro-images fit onto the sensor, however, in turn, their resolution increases. This phenomenon illustrates that the spatio-angular trade-off is linked to the position of the exit pupil of the main lens.

\section{A New Reference Point Detector}

As discussed previously, finding the brightest point (BP) of the sub-image is not a reliable calibration scheme in the general case. The physical method of [27] finds the ground truth points (GT) but necessitates special experimental procedures. Moreover, this method does not work for microlenses close to the image boundary since rays passing through the pupil center are vignetted. We therefore propose a new reference point detection scheme (Ours).

From an optical perspective, the round shape of the subimages in the center of the sensor is the consequence of the roundness of the exit pupil which primarily defines the shape of the light cone that reaches each of the microlenses even though the microlenses do not necessarily conjugate the exit pupil with the micro-image plane. Due to vignetting, the sub-images near the image boundary exhibit the well known cat's eye shape that is the result of the intersection of two or more circular apertures of different centers and diameters. This implies that part of the vignetted sub-images' shapes is due to the exit pupil.

Thus, we propose a new method to estimate the ground truth point (GT) which is to fit circles to the outline of the subimages. This step is performed by the imfindcircles function from MATLAB which uses an algorithm that is based on the circular Hough transform. The function takes as input the white image and a radius range. We estimate the average radius of the circles using the Fourier transform of the white image [26] and we set the radius range at $\pm 25 \%$ this estimate.

In the case of cat's eye sub-images, we typically find multiple circles (usually the pupil + another aperture) and thus multiple candidate centers. We use correctly detected single circles from the central sub-images to establish statistics on the circle radius in order to set a more narrow radius threshold to filter out unwanted candidates. In addition, since the grid layout of the MLA is usually regular (rectangular or hexagonal), we also estimate the position of the center from neighboring confirmed candidates and pick the closest candidate center to the estimate. This step is often necessary for sub-images on the sensor boundary and is robust in case of grid distortion caused by the optics (e.g. relay optics as in our custom built microscope, Sect. IV].

\section{E. Comparative Evaluation of Reference Point Detection Schemes}

To illustrate the different techniques and to compare to our own, we have computed the white image of the setup from Fig. 2a in Fig. 3 We also show the statistics of the error of these techniques in Table II

a) Orthogonal projection (OP, blue $\times$ marker) and brightest point (BP, green $\square$ markers): We observe that the brightest point (BP) of the sub-images matches the orthogonal projection (OP) of the microlens centers well in the area close 
TABLE II

STATISTICS OF THE ERROR OVER ALL SUB-IMAGES OF THE POINT ESTIMATION METHODS FOR THE MOCKUP SYSTEM. THE ERROR IS COMPUTED AS THE DISTANCE BETWEEN THE ESTIMATED POINT AND THE GROUND TRUTH (GT). VALUES ARE IN PIXELS

\begin{tabular}{|l|c|c|c|}
\hline Method & Mean Value & Standard Deviation & Root Mean Square \\
\hline (Ours) & $\mathbf{0 . 7 8}$ & $\mathbf{0 . 3 7}$ & $\mathbf{0 . 8 6}$ \\
(WA) & 1.23 & 0.57 & 1.35 \\
(BP) & 3.62 & 1.15 & 3.79 \\
\hline
\end{tabular}

to the center of the sensor. As such, we can infer that the (BP) techniques can be used to extract the true microlens spacings when they are unknown. At the border of the image, though, these microlens centers do not correspond to the intensity peaks anymore because of the perspective shift of the sub-images, rendering the detection of the microlens grid parameters impractical. In our mockup system, the distance between neighboring ground truth points is 16 pixels whereas the distance between neighboring microlens centers is equal to 14 pixels. In a real system, the perspective shift is smaller to those shown because of the larger exit pupil distance. The majority of the grid is usually fit to the center lenslets that are unbiased, resulting in similar effects at the image boundary as shown in Fig. 3 (OP).

b) Weighted Average (WA, purple $\diamond$ markers): These points provide a better estimate of the ground truth but still fail for vignetted sub-images because the shape of the sub-images becomes asymmetric on the image border and is not centered on the ground truth.

c) Ground truth (GT, brown + markers) and circle fitting (Ours, yellow o markers): Our circle based method is robust to vignetting and the only one that matches the ground truth for all sub-images. This accordance between the ground truth and our detection method validates the ability of our vignetting model to simulate actual white images of the light field camera system because the perspective projections of the exit pupil center match the detected sub-image center that was computed without knowledge of the optical system parameters.

\section{VIGNETTING EFFECTS ON THE PROPERTIES OF LIGHT FIELD CAMERAS}

The previous discussion is on the pixel level, the overall effect is better visible when the system is interpreted as an equivalent camera array as introduced in [1]. In this section, we study the effects of vignetting on the properties of the ECA corresponding to the previously simulated mockup optical system. We continue by applying the ECA model with and without vignetting to a real experiment using our custom light field microscope system with known first-order properties.

\section{A. Phase space analysis of ECA vignetting}

The ECA model is a first-order optical model [1] that represents many different light field camera designs with an equivalent camera array in object space. The motivation behind the model is to set a common ground for a comparison of the optical properties of various light field camera architectures. The common representation, the equivalent camera array, consists of a set of virtual cameras, each with its own virtual aperture and virtual sensor that lie in object space. This model enables to define per-camera properties such as the viewing direction, the angle of view and the depth of field as well as field properties such as the baseline, and the spatial and longitudinal accuracies at any point in the object space.

These properties can be understood by looking directly at the phase space footprint of the camera. We take the mockup system from Fig. 2a as an illustrative example. In the 2D phase space diagram Fig. 2b, each cell in blue corresponds to the ray bundle passing through the apertures of one microlens/pixel pair. Each column corresponds to a different view which is called a virtual camera in the ECA model. All columns contain the same number of cells because the same number of pixels is selected naively behind each microlens without any vignetting consideration. As defined in [1], the vertical extent of a column between the $\times$-markers gives the angle of view ${ }^{1}$ of the corresponding virtual camera. The midpoint marked by *-markers gives the viewing direction. For the definition of the other optical properties, we refer to [1].

The black outline delimits the region for which rays are not stopped by the apertures of the components of the main objective, i.e. the two lenses and the diaphragm. Within our vignetting model, it corresponds to Equation 6 excluding from the intersection the indicator functions of the pixel $\chi_{0}^{S o}$ and of the microlens aperture $\chi_{1}^{S o}$ which are already used to produce the blue cells. In the 2D phase space diagram, the indicator function of one aperture is equal to 1 in the region between two parallel lines, so the intersection of the indicator functions of two apertures in different planes produces a rhombic cell like the one of a pixel/microlens pair. For the three apertures of the main objective, it creates the hexagonal black shape. In the true 4D phase space, the pair of parallel lines visualizing the aperture indicator function is replaced by a pair of parallel 2D planes.

In order to get the true vignetted cells, we intersect the phase space region of the main objective with that of the blue cells resulting in the red cells. We also observe the creation of red cells where blue cells were not present in the unvignetted case. This is due to a different assignment of pixels to microlenses caused by the perspective shift of the sub-images. Overall, we obtain modified values for the angle of view and the viewing direction of the virtual camera, respectively the vertical extent and midpoint of the vertical columns of the red cells. Most of the other properties of the equivalent camera array are affected as well, as we show in the following on a real experiment.

\section{B. Vignetting in the light field microscope}

In order to validate our vignetting model experimentally, we have built a light field microscope according to the design introduced by [21]. The parameters of the simulation match those of the built microscope.

1) Setup: The light field microscope is composed of two main parts from each side of the MLA, see Fig. 6a

The magnifying part is made of the microscope objective and the tube lens and it conjugates the object plane with the

\footnotetext{
${ }^{1}$ This value describes the field of view of the virtual camera. We use the terminology of [1] for consistency.
} 


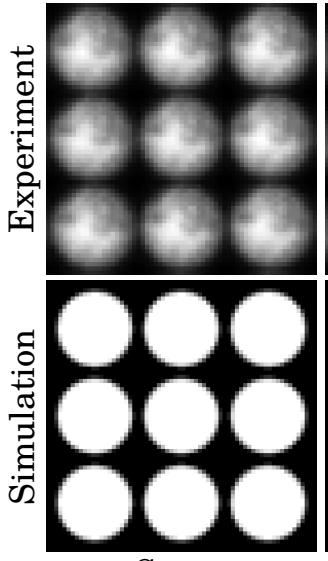

Center

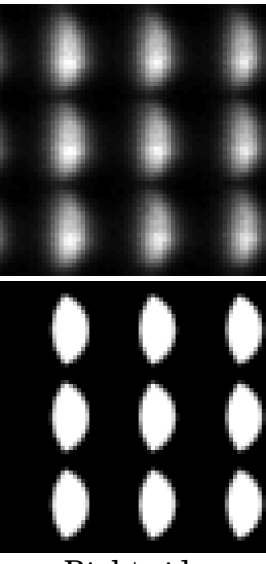

Right side
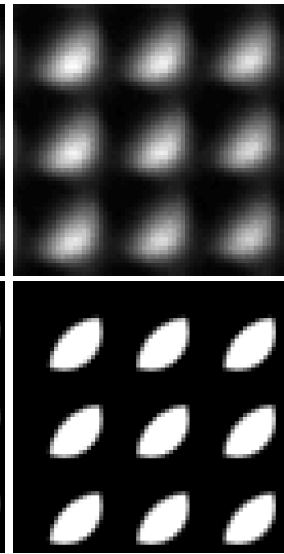

Bottom right corner

Fig. 4. Snapshots of the sub-images of the white image at different locations on the field for the real setup (top row) and the simulation (bottom row). The contrast of all the pictures has been normalized for better visualization. The side and corner pictures represent the sub-images at $80 \%$ of the field The shape of the sub-image continue to reduce along with their increasing distance from the sensor center.

plane containing the MLA. The magnification is performed by an infinity-corrected objective with a factor of $20 \times$ and a numerical aperture of 0.5 together with a tube lens with a focal length of $200 \mathrm{~mm}$ and a diameter of $30 \mathrm{~mm}$. The image-side $\mathrm{f}$-number of this optical system is 20 .

The relay part is made of a field lens and the camera objective. Its task is to conjugate the image plane of the microlenses (one microlens focal length away from the MLA plane) with the sensor plane. The field lens is a doublet with a focal length of $75 \mathrm{~mm}$ and a diameter of $48 \mathrm{~mm}$ placed close to the back (flat side) of the microlens array. A Canon camera with an objective lens of focal length $100 \mathrm{~mm}$, operating in a $1: 1$ macro configuration at $f / 2.8$ is used as the sensor.

Regarding the MLA, each microlens is plano-convex and has a focal length of $2.1 \mathrm{~mm}$. Its aperture is a square with a side length of $100 \mu \mathrm{m}$, hence, an f-number of 21 . The sensor of the Canon 5D Mark II camera has $5616 \times 3744$ pixels and a total size of $36 \mathrm{~mm} \times 24 \mathrm{~mm}$. The pixel side length of the sensor is $6.41 \mu \mathrm{m}$. In this configuration, the role of the virtual cameras usually associated to the images of the microlenses can be attributed to the images of pixels as discussed in [1].

2) White image: On the raw image, the micro-images are disks with a diameter of 21 pixels arranged in a rectangular grid. It means that the magnification of the relay part is 1.35 . The number of microlenses covering the sensor is $270 \times 180$. In Fig. 4, we show snapshots of the white images for the real setup and the simulation. The main difference is the unevenness of the intensity distribution. This effect is mostly the consequence of some lenses not being clean and the scene not being completely Lambertian. In the simulated pictures, the rays reach the pixels with an angle close to the normal because the f-number is high so the pixels inside the sub images have almost the same brightness. Note the close similarity of the sub-image shapes, the blur being due to unmodeled aberrations.

3) Comparison between the unvignetted and vignetted cases: Fig. 5 shows the properties of the ECA in the un- vignetted and vignetted cases. In the following, we analyze each property. The virtual cameras are denoted by their index, 0 being attributed to the camera on the optical axis. They are spaced symmetrically on both sides of the optical axis in both cases. In Figs. 5d to 51, the vertical red line indicates the plane of the virtual cameras. The vertical dotted blue line indicates the plane of the virtual sensor which is also the image of the microlenses and the object plane. The black dotted line on each side denotes the limits of the depth of field $(180 \mu \mathrm{m})$. The depth of field has a value of $890 \mu \mathrm{m}$. It is not modified by vignetting because the distance along the optical axis between the virtual camera (red line) and the virtual pixels (blue line) as well as their respective apertures $(45 \mu \mathrm{m}$ and $4.5 \mu \mathrm{m})$ remain unchanged.

a) Number of virtual cameras and pixels (Fig. 5a): In the unvignetted case, the number of cameras is 21 which corresponds to the number of pixels directly behind a microlens: the ratio of the microlens to the pixel extents corrected by the magnification factor of the relay part. The number of virtual pixels is equal to the number of microlenses: 270 .

In the vignetted case, the number of cameras reaches 49 and the number of virtual pixels is no longer constant nor equal to the unvignetted case. Three main parts can be observed on the curve separated by two pairs of symmetric inflection points (at camera indices -8 and 8 for the first pair and -15 and 15 for the second). These changes in the curve slope are due to vignetting by two different apertures at different positions of the field: the apertures of the microscope objective and the field lens. The lower number of pixels for the central part is due to the vignetting caused by the tube lens. The aperture of the microscope objective mainly controls the number of virtual cameras and the field lens reduces this number a bit more. The linear nature of the parts of the curve is explained by the pixel number being the count of a $1 \mathrm{D}$ cross section of the virtual sensor and not the total number of pixels in the sub-image.

b) Viewing direction (Fig. 5b): In the unvignetted case, all the virtual cameras are looking at the same position at the center of the MLA image. In the vignetted case, though, asymmetrical vignetting causes the point of observation of the cameras to shift away along the optical axis. We again observe the inflection points in the same position as in Fig. 5a

c) Angle of view (Fig. 5b): In the unvignetted case, all the cameras share the same object field whereas, in the vignetted case, the decrease of the curve on the edges is primarily due to a decrease in the number of virtual pixels.

d) Baseline (Fig. 5d 5g 5j): The baseline value associated to a point in object space is the maximum distance between virtual cameras that see the point in their field of view. In the depth of field region, the baseline stays the same at around $1 \mathrm{~mm}$ for a point on the optical axis because the same number of cameras covers this part in both cases. However, the shift and size of the field of view of the virtual cameras changes the values of the baseline in the rest of the field. The extent of the region seen before the object plane (vertical dotted blue line) is larger in the vignetted case and its size decreases behind the object plane.

e) Transversal accuracy (Fig. 5e, 5h, 5k): For similar reasons to the unchanged depth of field, the variation of the 


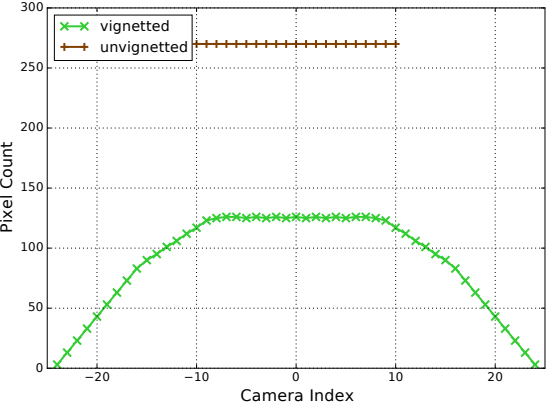

(a) Number of pixels per camera

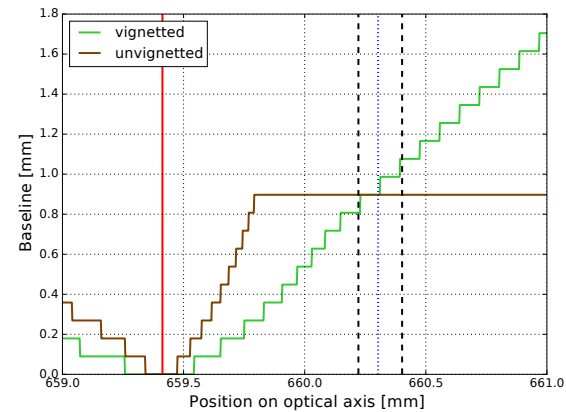

(d) Baseline on axis

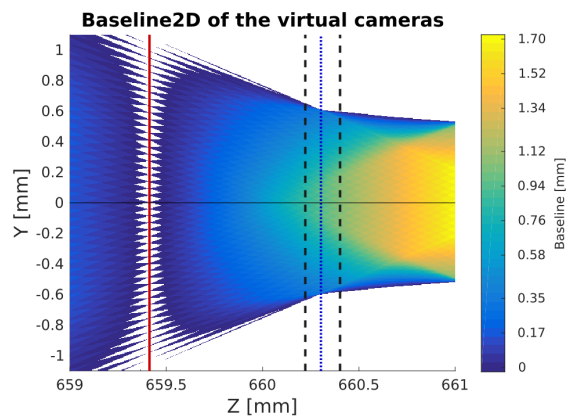

(g) Vignetted Baseline on field

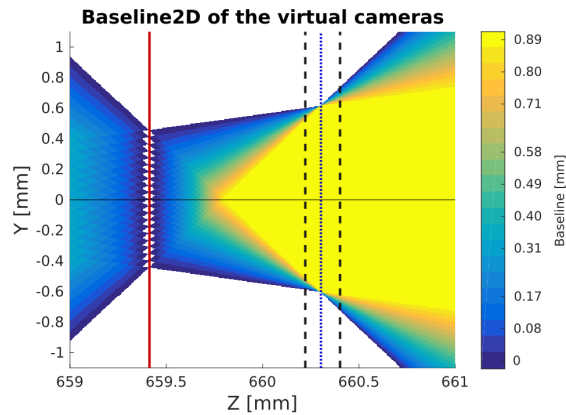

(j) Unvignetted Baseline on field

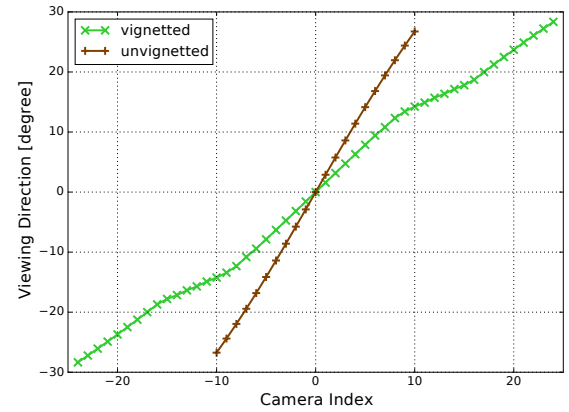

(b) View direction

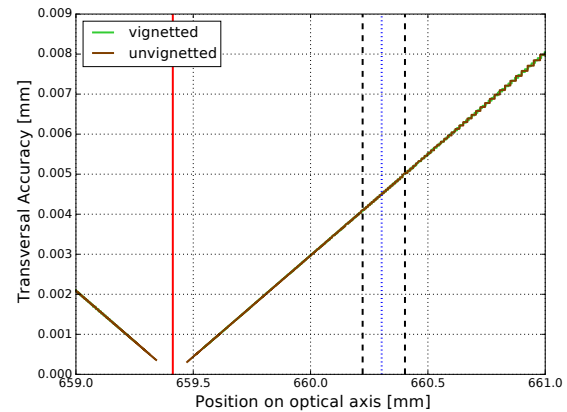

(e) Transversal Accuracy on axis

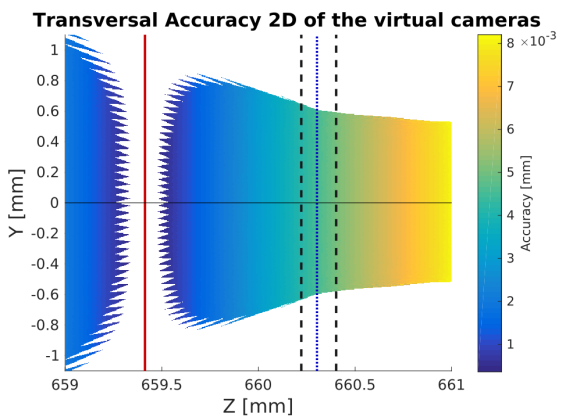

(h) Vignetted Transversal Accuracy on field

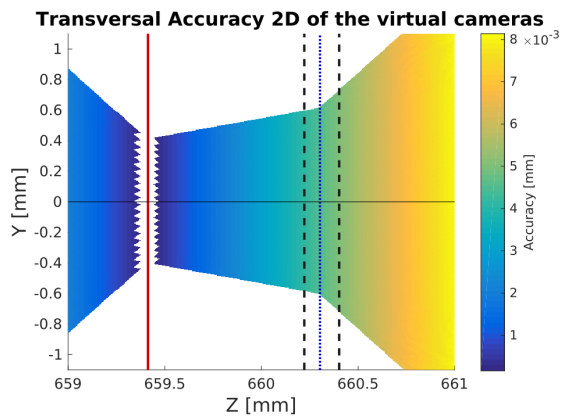

(k) Unvignetted Transversal Accuracy on field

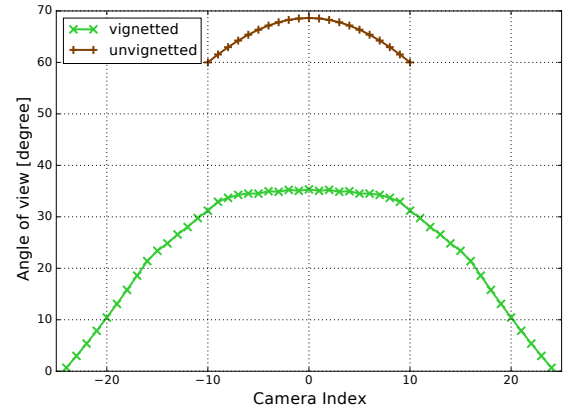

(c) Angle of view

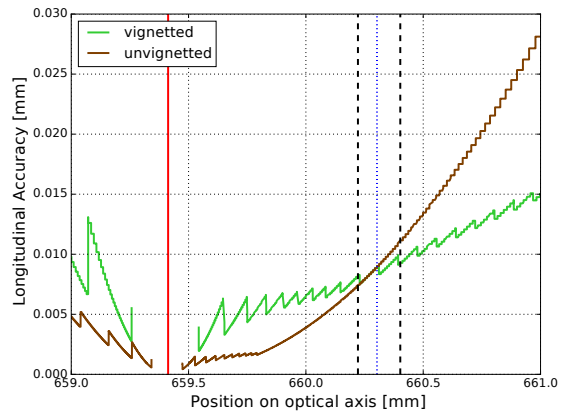

(f) Longitudinal Accuracy on axis

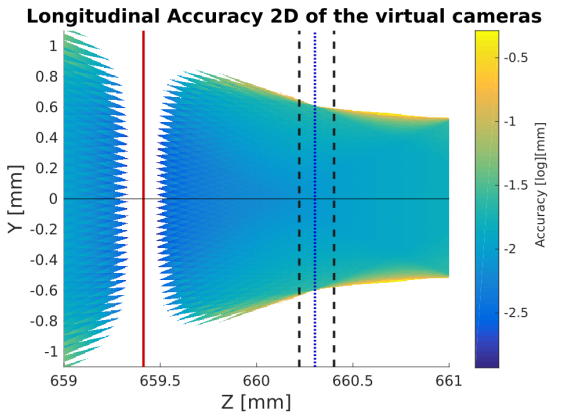

(i) Vignetted Longitudinal Accuracy on field

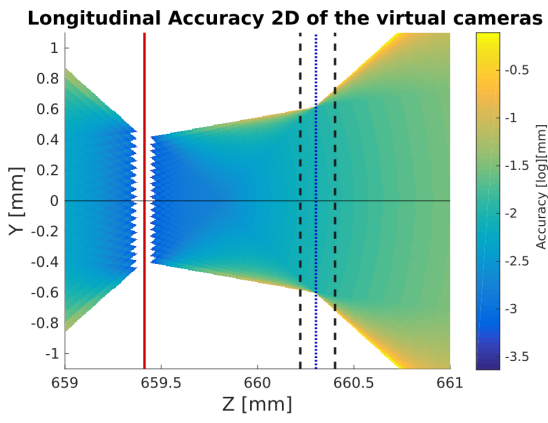

(1) Unvignetted Longitudinal Accuracy on field

Fig. 5. Virtual camera properties and field properties of the ECA of the microscope setup. For details and interpretation, see the main text.

transversal accuracy remains unchanged. The extent of the field coverage follows that of the baseline. The transversal accuracy is between $4 \mu \mathrm{m}$ and $5 \mu \mathrm{m}$ in the depth of field range.

f) Longitudinal accuracy (Fig. 5f] 5i] 57): The color scale of these figures is the $\log 10$ of the actual value and smaller numbers are better, indicating the extent of a depth-resolvable region. The longitudinal accuracy tends to increase with the distance to the virtual camera (vertical red line) and it jumps down with each increase in the baseline. In the unvignetted case, the baseline reaches its maximum value early whereas in the vignetted case, the baseline is lower at first but continues to increase because of the larger number of cameras. Thus, in front of the object plane (vertical dotted blue line), the accuracy is higher in the unvignetted case because the baseline is lower. After the object plane, the baseline is higher, so the accuracy is lower than for the unvignetted case. In the depth of 
field range, the longitudinal accuracy is between 7 and $9 \mu \mathrm{m}$. g) Summary: Vignetting affects mostly the number of virtual cameras and their pixel count which greatly modifies their angle of view and viewing direction. In the depth of field region, the baseline stays quite stable and is less affected by vignetting than what could have been expected. The longitudinal accuracy even improves slightly and the transversal accuracy remains almost unchanged.

4) Experimental results: We used a 1951 USAF test chart in Fig. $6 b$ to evaluate the performance of the system. The system reaches a resolution of $114 \mathrm{lp} / \mathrm{mm}$ for a magnification of the first part of the system of 22.2. The size of a microlens in object space being $4.5 \mu \mathrm{m}$, this is at the upper limit of the expected performance, see Fig. 5e. The overall aberrations of the views are low and constant even on the side views but anisotropic. Visual differences between (BP) and (Ours) techniques can be noted. The contrast and the sharpness of the patterns and number are better in (Ours) for both the top and right views. Moreover, the resampling artifacts are enhanced in the (BP) decoding.

Fig. 7 shows a sheet of optical paper and its reconstructed depth map estimated using the algorithm of [19]. The fiber structures of the optical paper are wide enough to be resolved correctly. In this single layer, the depth difference between the closest and furthest fiber is approximately $150 \mu \mathrm{m}$ which is close to the simulated depth of field. The longitudinal accuracy is estimated as approximately $10 \mu m$ (evaluating the color scale) which matches the simulation Fig. 5f. Strong vignetting on the side of the image and a lack of fibers are causing the deep hole on the top right corner.

\section{CONCLUSiOn \& Future WORKS}

We have introduced a vignetting model for light field cameras that is using their optical system layout. We have described this new model using set theory in phase space in a camera model independent way. It is based on the imaging of the system apertures to object space followed by an intersection of their indicator functions. With this vignetting model, we have simulated the distribution of light intensity on the sensor from a flat field scene (the white image) that is usually used in light field camera calibration. We have assessed that the ground truth center of the sub-images to extract the light field from the sensor is the image of the exit pupil of the main lens. Moreover, we have observed that the state of the art detection schemes present an inherent bias so we have proposed a new one based on circle detection to retrieve these reference points in the case that no optical system model is available. This new method is robust to the change of sub-image shape caused by vignetting and performs better than other methods from the literature. We have shown how vignetting modifies the pixel assignment of virtual cameras and, consequently, changes their viewing direction, their field of view and the light field sampling in general. Finally, we have built our own light field microscope setup to verify the validity of our model.

We have seen that our study of vignetting allows to compute the light field elementary kernels in phase space. This information would certainly benefit light field reconstruction processes and we leave this task as future work. We could also consider to apply our model to improve light field projection devices such as auto-stereoscopic displays. More generally, an inverse model that would help conceive a light field camera from a list of its object side optical parameters is still lacking.

\section{ACKNOWLEDGMENT}

This work was supported by the ANR ISAR project. The authors would like to thank the Institut National de Recherche en Informatique et Automatique (INRIA) Bordeaux SudOuest as well as the Laboratoire Photonique, Numérique et Nanosciences (LP2N) at which part of this work were carried out.

\section{REFERENCES}

[1] L. Mignard-Debise, J. Restrepo, and I. Ihrke, "A unifying first-order model for light-field cameras: the equivalent camera array." IEEE Transactions on Computational Imaging, 2017.

[2] G. Lippmann, "Epreuves reversibles donnant la sensation du relief," $J$. Phys. Theor. Appl., vol. 7, no. 1, pp. 821-825, 1908.

[3] E. H. Adelson and J. Y. A. Wang, "Single lens stereo with a plenoptic camera," IEEE Transactions on Pattern Analysis \& Machine Intelligence, no. 2, pp. 99-106, 1992.

[4] R. Ng, M. Levoy, M. Brédif, G. Duval, M. Horowitz, and P. Hanrahan, "Light field photography with a hand-held plenoptic camera," Computer Science Technical Report CSTR, vol. 2, no. 11, 2005.

[5] A. Lumsdaine and T. Georgiev, "The focused plenoptic camera," in Computational Photography (ICCP), 2009 IEEE International Conference on. IEEE, 2009, pp. 1-8.

[6] B. Wilburn, N. Joshi, V. Vaish, M. Levoy, and M. Horowitz, "Highspeed videography using a dense camera array," in Computer Vision and Pattern Recognition, 2004. CVPR 2004. Proceedings of the 2004 IEEE Computer Society Conference on, vol. 2. IEEE, 2004, pp. II-294.

[7] B. Wilburn, N. Joshi, V. Vaish, E.-V. Talvala, E. Antunez, A. Barth, A. Adams, M. Horowitz, and M. Levoy, "High performance imaging using large camera arrays," ACM Transactions on Graphics (TOG), vol. 24, no. 3, pp. 765-776, 2005.

[8] K. Venkataraman, D. Lelescu, J. Duparré, A. McMahon, G. Molina, P. Chatterjee, R. Mullis, and S. Nayar, "Picam: An ultra-thin high performance monolithic camera array," ACM Transactions on Graphics (TOG), vol. 32, no. 6, p. 166, 2013.

[9] I. Light, "Light website," https://light.co/, 2017, accessed: 2017-08-21.

[10] T. Georgiev and C. Intwala, "Light field camera design for integral view photography," Adobe System, Inc, Tech. Rep., 2003.

[11] T. Yamamoto, M. Kojima, and T. Naemura, "Liflet: Light field live with thousands of lenslets," in ACM SIGGRAPH 2004 Emerging technologies. ACM, 2004, p. 16.

[12] K. Ueda, T. Koike, K. Takahashi, and T. Naemura, "Adaptive integral photography imaging with variable-focus lens array," Proc SPIE: Stereoscopic Displays and Applications XIX, pp. 68 031A-9, 2008.

[13] Y. Taguchi, A. Agrawal, A. Veeraraghavan, S. Ramalingam, and R. Raskar, "Axial-cones: Modeling spherical catadioptric cameras for wide-angle light field rendering," ACM Trans. Graph., vol. 29, no. 6, pp. 172-1, 2010.

[14] J. Unger, A. Wenger, T. Hawkins, A. Gardner, and P. Debevec, "Capturing and rendering with incident light fields," DTIC Document, Tech. Rep., 2003.

[15] M. Levoy, B. Chen, V. Vaish, M. Horowitz, I. McDowall, and M. Bolas, "Synthetic aperture confocal imaging," in ACM Transactions on Graphics (ToG), vol. 23, no. 3. ACM, 2004, pp. 825-834.

[16] M. Fuchs, M. Kächele, and S. Rusinkiewicz, "Design and fabrication of faceted mirror arrays for light field capture," in Computer Graphics Forum, vol. 32, no. 8. Wiley Online Library, 2013, pp. 246-257.

[17] I. Ihrke, I. Reshetouski, A. Manakov, A. Tevs, M. Wand, and H.-P. Seidel, "A kaleidoscopic approach to surround geometry and reflectance acquisition," in Computer Vision and Pattern Recognition Workshops (CVPRW), 2012 IEEE Computer Society Conference on. IEEE, 2012, pp. 29-36.

[18] I. Reshetouski, A. Manakov, H.-P. Seidel, and I. Ihrke, "Threedimensional kaleidoscopic imaging," in Computer Vision and Pattern Recognition (CVPR), 2011 IEEE Conference on. IEEE, 2011, pp. 353360 


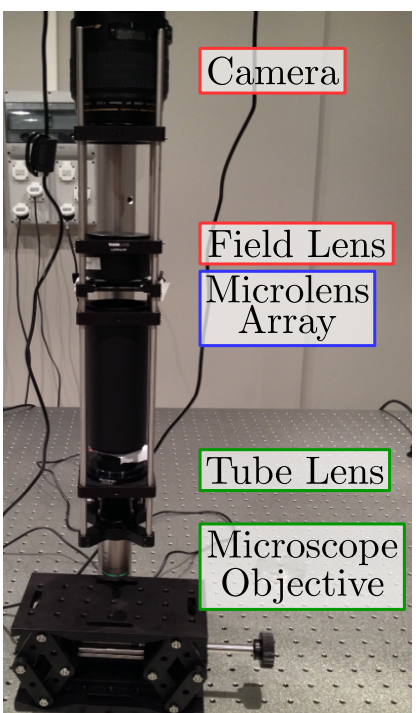

(a) LFM Setup
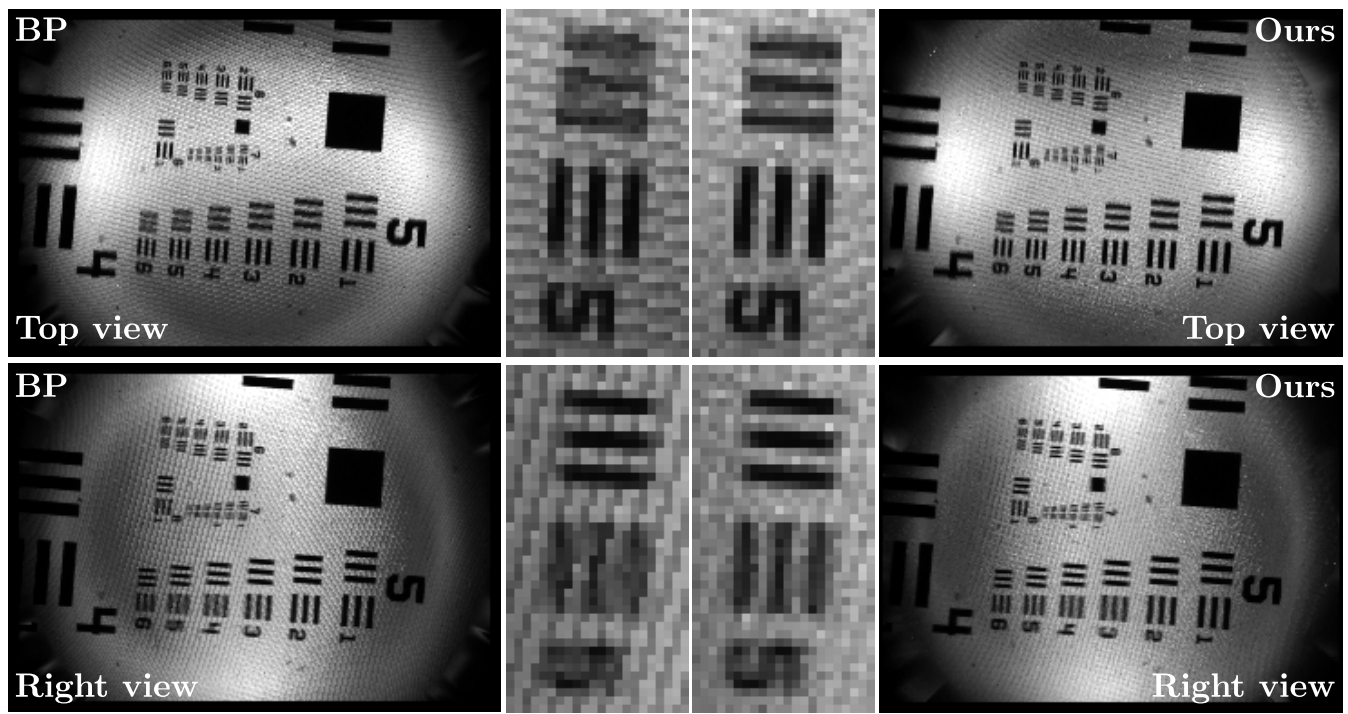

(b) Views of the USAF calibration target

Fig. 6. (a) Setup of the light field microscope. (b) Extreme top and right views of the USAF calibration target using two light field calibration techniques: (BP) on the left and (Ours) on the right. The intensity of the pictures has been leveled to the intensity of their respective center view. The insets show a zoomed portion of the 5-5 bars of the USAF chart used for qualitative comparison of the techniques. The extracted views (BP) have stronger blur and sampling artifacts as compared to (Ours). Note that the experiment was performed in the object plane where incorrect view assignments have the least effect. The results therefore present an upper bound on the achievable quality using the inaccurate calibration.
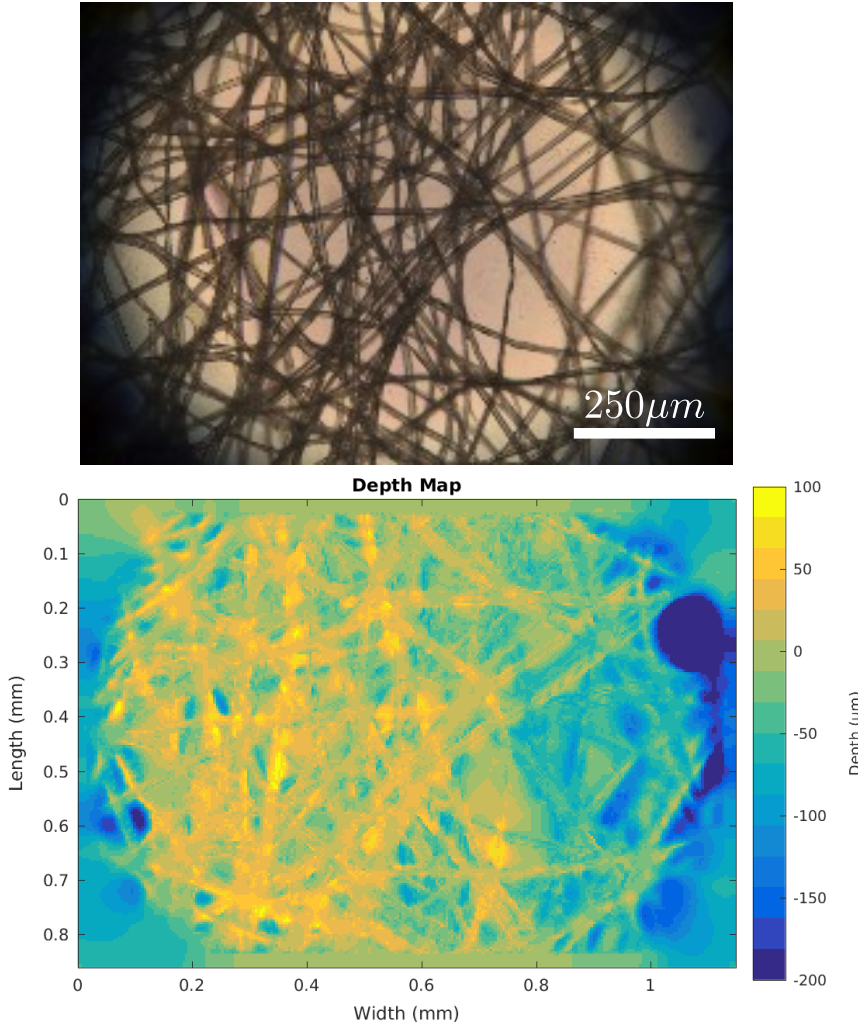

Fig. 7. Sample of a sheet of optical paper and its reconstructed depth map.

[19] A. Manakov, J. Restrepo, O. Klehm, R. Hegedüs, H.-P. Seidel, E. Eisemann, and I. Ihrke, "A reconfigurable camera add-on for high dynamic range, multispectral, polarization, and light-field imaging," ACM Trans. on Graphics (SIGGRAPH'13), vol. 32, no. 4, pp. 47:1 - 47:14, 2013.

[20] S. Pacheco and R. Liang, "Snapshot, reconfigurable multispectral and multi-polarization telecentric imaging system," Optics express, vol. 22, no. 13, pp. 16377-16385, 2014.

[21] M. Levoy, R. Ng, A. Adams, M. Footer, and M. Horowitz, "Light field microscopy," in ACM Transactions on Graphics (TOG), vol. 25, no. 3 .
ACM, 2006, pp. 924-934.

[22] T. Georgiev, C. Intwala, S. Babakan, and A. Lumsdaine, "Unified frequency domain analysis of lightfield cameras," Computer VisionECCV 2008, pp. 224-237, 2008.

[23] C. Hahne, A. Aggoun, S. Haxha, V. Velisavljevic, and J. C. J. Fernández, "Light field geometry of a standard plenoptic camera," Optics Express, vol. 22, no. 22, pp. 26659-26673, 2014.

[24] C.-K. Liang and R. Ramamoorthi, "A light transport framework for lenslet light field cameras," ACM Transactions on Graphics (TOG), vol. 34, no. 2, p. 16, 2015.

[25] M. Damghanian, R. Olsson, and M. Sjostrom, "Extraction of the lateral resolution in a plenoptic camera using the spc model," in $3 D$ Imaging (IC3D), 2012 International Conference on. IEEE, 2012, pp. 1-5.

[26] D. Cho, M. Lee, S. Kim, and Y.-W. Tai, "Modeling the calibration pipeline of the lytro camera for high quality light-field image reconstruction," in Computer Vision (ICCV), 2013 IEEE International Conference on. IEEE, 2013, pp. 3280-3287.

[27] C. Thomason, B. Thurow, and T. Fahringer, "Calibration of a microlens array for a plenoptic camera," in 52nd Aerospace Sciences Meeting: American Institute of Aeronautics and Astronautics, 2014, p. 0396.

[28] D. G. Dansereau, O. Pizarro, and S. B. Williams, "Decoding, calibration and rectification for lenselet-based plenoptic cameras," in Computer Vision and Pattern Recognition (CVPR), 2013 IEEE Conference on. IEEE, 2013, pp. 1027-1034.

[29] O. Johannsen, C. Heinze, B. Goldluecke, and C. Perwaß, "On the calibration of focused plenoptic cameras," in Time-of-Flight and Depth Imaging. Sensors, Algorithms, and Applications. Springer, 2013, pp. 302-317.

[30] C. Heinze, S. Spyropoulos, S. Hussmann, and C. Perwass, "Automated robust metric calibration of multi-focus plenoptic cameras," in Instrumentation and Measurement Technology Conference (I2MTC), 2015 IEEE International. IEEE, 2015, pp. 2038-2043.

[31] D. C. O'Shea, "Elements of modern optical design," 1985.

[32] L. Mignard-Debise, "Tools for the paraxial optical design of light field imaging systems," Theses, Université de Bordeaux, Feb 2018. [Online]. Available: https://tel.archives-ouvertes.fr/tel-01764949

[33] J. Chaves, Introduction to nonimaging optics. CRC press, 2008.

[34] M. Schambach and F. P. Leon, "Algorithms for microlens center detection," in Forum Bildverarbeitung 2018. KIT Scientific Publishing, 2018, p. 229.

[35] Y. Wang, J. Qiu, C. Liu, D. He, X. Kang, J. Li, and L. Shi, "Virtual image points based geometrical parameters calibration for focused light field camera," IEEE Access, vol. 6, pp. 71 317-71 326, 2018. 